\title{
Nomenclature
}

$\rho$

$U_{\infty}$

$U$

$u_{i}$

$u_{o}$

$R, D$

$c$

$n_{b}$

$\sigma$

$T$

$N$

$C_{F_{n}}$

$C_{F_{t}}$

$\xi$

$S_{x}$

$S_{y}$

$\omega$

$\Omega$

$C_{P}$

\section{Flow Physics and Performance of Vertical Axis Wind Turbine Arrays}

\author{
Karthik Duraisamy, \\ Assistant Professor, Department of Aerospace Engineering, \\ University of Michigan, Ann Arbor, MI, \\ Vinod Lakshminarayan, \\ Post Doctoral Fellow, Department of Aeronautics and Astronautics, \\ Stanford University, Stanford, CA
}

\begin{abstract}
Modern wind farms employing horizontal-axis wind turbines (HAWTs) require significant turbine separation to avoid aerodynamic interference, leading to inefficiencies in the use of wind resources and land area. On the other hand, arrays of vertical axis wind turbines (VAWTs) have the potential to achieve higher power output per unit land area because of their smaller footprint. Recent studies by Dabiri ${ }^{1}$ and co-workers have indicated that additional benefits can be obtained by optimizing the placement and the direction of rotation of VAWTs. The focus of the current work is to study the flow physics and performance of VAWT arrays using numerical simulations based on the Reynolds Averaged Navier Stokes equations. The results show that the majority of the wake deficit is confined to a few diameters downstream of a single VAWT and its spatial extent is seen to diminish with increased rotational speed. The extent of wake deficit extends further downstream by placing multiple VAWTs in a single column, irrespective of the direction of rotation of individual turbines. The aerodynamic interference between turbines gives rise to regions of excess momentum between the turbines, which leads to a significant augmentation up to twice that of an isolated turbine. Studies of VAWTs arranged in multiple columns show that the downstream columns can actually be more efficient than the leading column when spaced optimally.
\end{abstract}

Density of freestream flow

Velocity of freestream flow

Mean streamwise velocity

Self-induced velocity

Induced velocity due to other turbines

Radius and Diameter of Turbine

Chord of blade airfoil section

Number of blades

Turbine solidity $n_{b} c / \pi D$

Tangential force per unit span

Normal force per unit span
Normal Force Coefficient $\frac{N}{\frac{1}{2} \rho_{\infty} c U^{2}}$

Azimuthal blade rotation angle

Horizontal separation distance between turbine axes

Vertical separation distance between turbine axes

Rotation rate

Tip speed ratio (TSR) $U_{\infty} / \omega R$

Power Coefficient $\frac{T \omega R}{\frac{1}{2} \rho_{\infty} D U_{\infty}^{3}}$ 


\section{Introduction}

Reducing the cost and footprint of wind turbines is critical to ensure the competitiveness of wind energy with more conventional sources of energy. The most established method of extracting energy from the wind involves the horizontal axis wind turbine (HAWT). The performance of an isolated HAWT is limited by the theoretical efficiency of approximately $59.3 \%$ of the energy of air passing through the swept area as stated by the well-known Betz limit. When operated as part of a wind farm, additional power losses due to aerodynamic interference between wind turbines dictates the spacing between wind turbines. The requirement of having to space turbines 8-10 diameters from each other, to minimize detrimental effects of aerodynamic interference, ${ }^{2}$ has been recognized to result in a wind farm productivity of less than 3 Watts per square meter ${ }^{3}$ of land area covered by the wind farm in many locations. While the land between the turbines can sometimes have incidental uses such as farming, the areas with greatest wind resources remain underutilized for energy generation. Furthermore, there is a limited availability of onshore sites that possess excellent wind resources and are not located prohibitively far from the power grid. It is essential that the wind resources in these locations are tapped as effectively as possible, as they are often more cost-effective than alternative installations that require extensive new grid infrastructure. Offshore wind energy installations would likewise benefit from more efficient use of those regions dedicated to wind energy production, as practical constraints limit the extent of coastal regions that can be dedicated to wind farms.

Vertical axis wind turbines (VAWTs), on the other hand, have the potential to improve this situation. Although an isolated VAWT is typically less efficient than a HAWT when comparing equivalent swept areas, the land footprint spanned by a VAWT can be less than that of a HAWT, since the rectangular swept area of a VAWT can be increased independently from its circular footprint. Further, recent field studies ${ }^{1,4}$ have suggested that additional gains may be expected via optimal placement and direction of rotation of VAWTs in a wind farm. These strategies aim to exploit wake dynamics that are unique to VAWTs. In particular, arrayed VAWTs can potentially achieve constructive aerodynamic interference that appears to be unattainable for HAWTs. There may also exist mechanisms to enhance the turbulent vertical flux of kinetic energy into VAWT arrays. The insensitivity of VAWTs to incident wind direction potentially enables them to function in higher levels of turbulence than conventional HAWTs.

The majority of recent work on VAWT arrays has used full-scale field measurements. While such realworld experiments are irreplaceable in furthering our capability to harness wind energy, the presence of a variety of complex phenomena and natural variabilities (such as wind direction, wind shear, terrain effects, measurement resolution, etc.) renders it difficult to isolate competing effects and clearly answer scientific questions. The objective of this work is thus to analyze the performance of arrays of VAWTs using a series of numerical simulations under controlled conditions with a focus on the underlying fluid dynamic phenomena. The broader goal of the paper is to determine the potential for significantly improved productivity of VAWTbased wind farms.

\section{Numerical Methodology}

The computations are performed using the solution of the compressible Unsteady Reynolds Averaged Navier-Stokes (URANS) equations. The OVERTURNS ${ }^{5,6}$ code which operates on structured overset meshes is employed for this purpose. An overset mesh approach is ideally suited for this application because each wind turbine blade can be represented using a single high quality mesh without the need to create matching interfaces with the rest of the computational domain. Additionally, this methodology allows for bodies in relative motion to be handled with ease. Figure 1 shows a sample overset mesh system involving three 2-bladed turbines.

The numerical method uses a third order accurate upwind scheme to compute the inviscid fluxes, while the viscous terms are discretized using second order accurate central differencing. Low Mach number preconditioning $^{7}$ is used to accelerate convergence within the context of dual-time stepping for implicit time integration. For turbulence closure, the Spalart-Allmaras ${ }^{8}$ turbulence model is employed.

\section{Validation and Verification}

In the past, the flow solver has been validated for a cyclo-rotor in hovering ${ }^{9}$ and forward flight conditions. ${ }^{10,11}$ More recently, the solver has been used to study the physics of small-scale VAWT with dynamic 


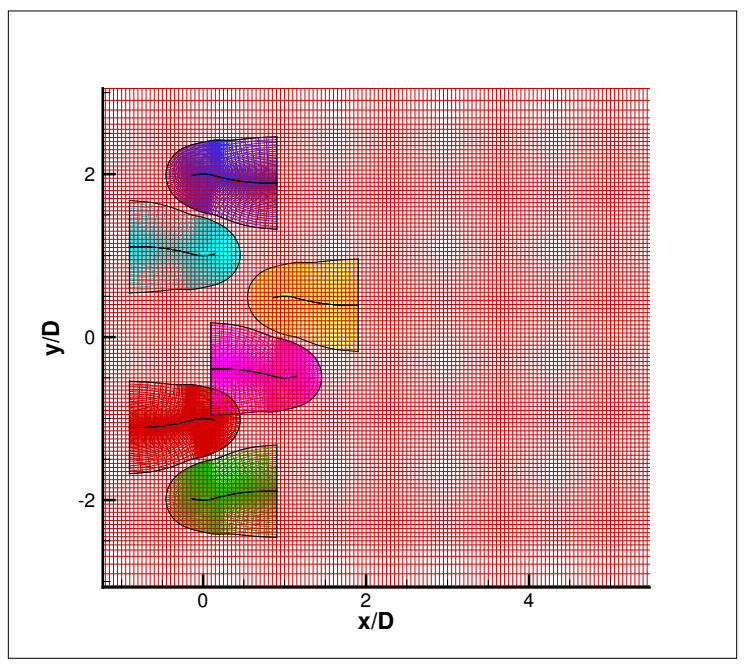

Figure 1. Sample overset mesh system for VAWT simulation: Background mesh (red) and blade meshes (black boundaries)

blade pitching. ${ }^{12}$ In this work, two additional validation cases are pursued. The first of these is a VAWT configuration and the second involves the simulation of counter-rotating cylinders, which is used as a proxy for a VAWT array in the absence of clean validation cases.

\section{A. VAWT validation}

The primary validation test case chosen for this work is based on an experimental setup of Oler et al. ${ }^{13}$ The setup consists of a one-bladed vertical axis wind turbine operating in a water tank. The blade uses a NACA 0015 airfoil and has a chord length of $15.24 \mathrm{~cm}$. The rotor diameter is $122 \mathrm{~cm}$, resulting in a chord to radius ratio $(c / R)$ of 0.25 . The rotor operates at a tip speed of $45.7 \mathrm{~cm} / \mathrm{s}$, yielding a chord-based Reynolds number of 67000 . Force measurements are available at three different tip to wind-speed ratio (TSR) of 2.5, 5.0 and 7.5. A schematic of the setup is shown in Fig. 2.

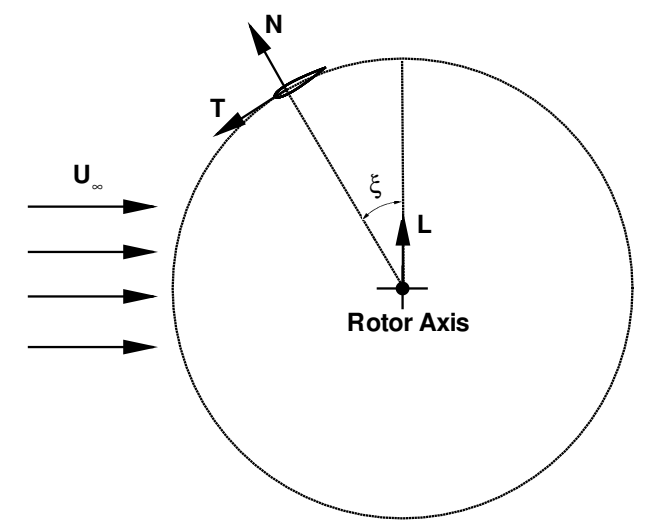

Figure 2. Flow schematic for vertical axis wind-turbine calculation.

Figure 3 compares the experimental and the predicted normal $\left(C_{F_{n}}\right)$ and the tangential $\left(C_{F_{t}}\right)$ force coefficient histories (non-dimensionalized by the freestream dynamic pressure and blade chord) as a function of the blade azimuth location for different TSRs. Although there are some quantitative differences, the results are satisfactory. One of the main reasons for the discrepancy is the assumption of two dimensionality in the simulation whereas the experiment used a short aspect ratio blade. Note that other geometric features such as the hub of the turbine and the connecting rod have also been ignored in the present simulations. 


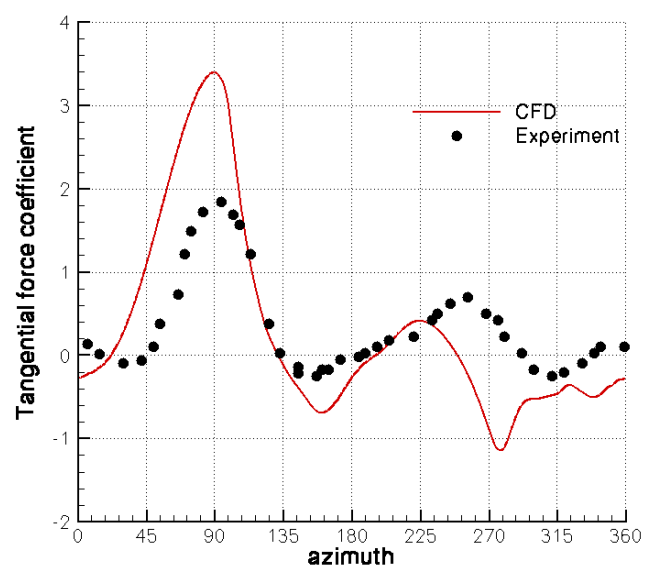

(a) $C_{F_{n}}$, TSR $=2.5$

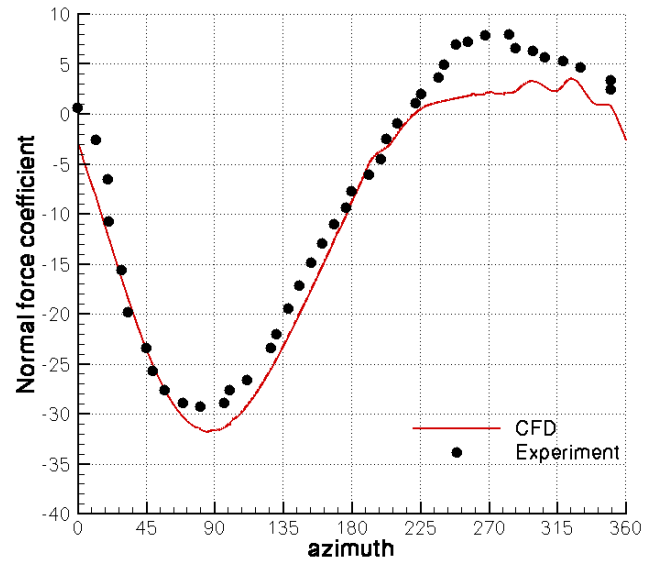

(c) $C_{F_{n}}, \mathrm{TSR}=5.0$

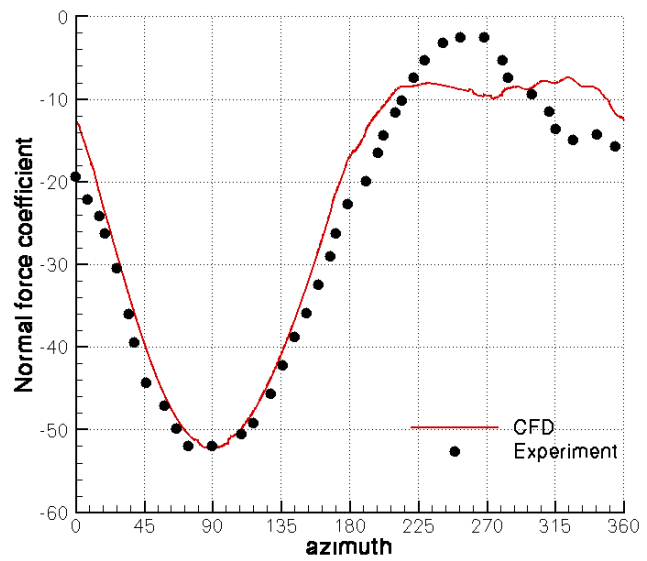

(e) $C_{F_{n}}, \mathrm{TSR}=7.5$

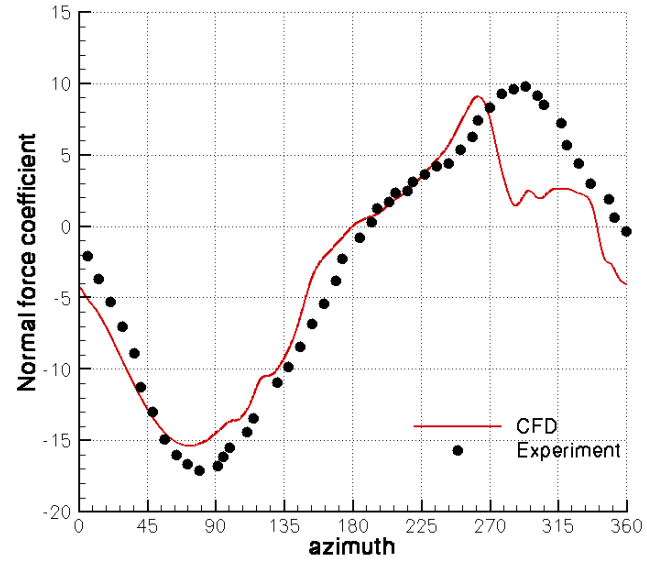

(b) $C_{F_{t}}$, TSR $=2.5$

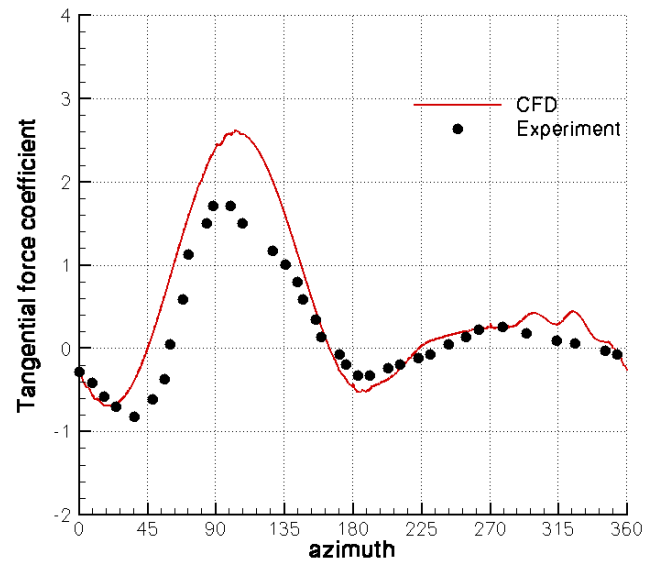

(d) $C_{F_{t}}$, TSR $=5.0$

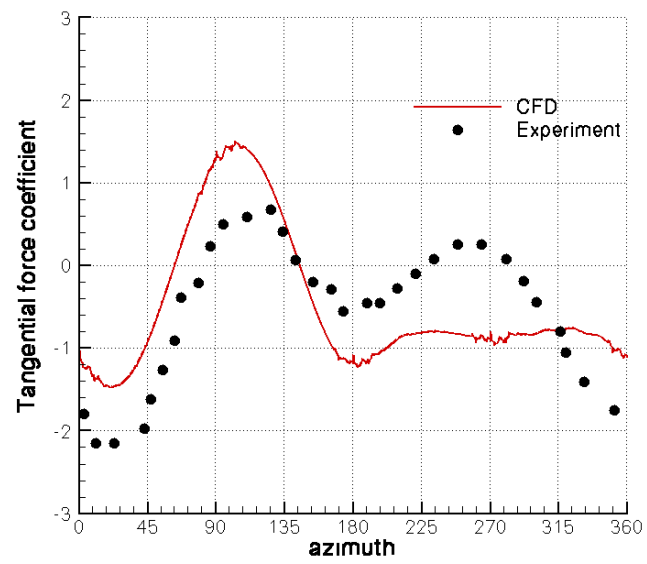

(f) $C_{F_{t}}, \mathrm{TSR}=7.5$

Figure 3. Comparison of normal and tangential force coefficient histories at different tip to wind-speed ratio for the Oler VAWT problem. 


\section{B. Two counter-rotating cylinders}

Next, the flow solver is validated in a laminar flow configuration past a counter-rotating cylinder pair (Fig. 4). The computation is performed at Reynolds number of 200 and Mach number of 0.05 . The distance between the cylinders, $g$ is set to $3 d$, where $d$ is the diameter of both the cylinders. Simulations are performed at different non-dimensional rotational frequencies, $\Omega=\frac{\omega d}{2 U}$, where $\omega$ is the dimensional rotational frequency and $U$ is the free-stream velocity.

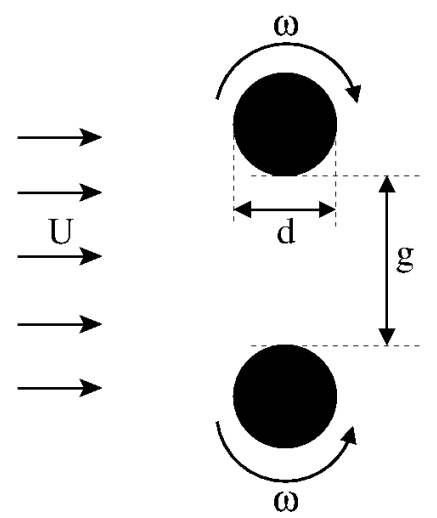

Figure 4. Schematic of doublet-like counter-rotating cylinder pair.

Figure 5 compares the streamlines and vorticity contours at different rotational speeds with the experimental flow visualization. ${ }^{14}$ Good qualitative agreement is noted between the computation and the experiment. Below the critical rotational speed of $\Omega=1.88$, stable vortex shedding is observed. As the rotational speed increases, the vortex strength decreases until the unsteady vortex wake is entirely suppressed. With a further increase in rotational speed, the extent of the wake behind the cylinders is decreased.

Figure 6 quantitatively compares the predicted time-averaged lift and drag coefficient on the upper cylinder at different rotational speeds with the computational results obtained by Chan et al. ${ }^{14}$ The excellent verification of the results thus provides confidence in the computational methodology to investigate VAWT flowfield arrays.

\section{The VAWT flow field}

In this section, isolated two and four-bladed VAWTs will be considered with the objective of studying the performance characteristics and the behavior of the wake. For all the cases considered henceforth in this work, the airfoil section is taken to be a NACA 0015 and the ratio of the blade chord to turbine radius is $c / R=0.314$. Two and four bladed turbines thus possess solidities $\sigma=\frac{n_{b} c}{2 \pi R}$ of 0.1 and 0.2 , respectively. The blade pitch angle is set to $0^{\circ}$. The Reynolds number of the freestream flow (based on the diameter of the VAWT) is taken to be $10^{5}$. Figure 7 shows contours of the time averaged wake deficit (normalized by the freestream velocity) for two and four-bladed VAWTs. The majority of the wake deficit is confined to a few diameters downstream of the VAWT and its spatial extent is seen to diminish with increased rotational speed as confirmed further in Figure 8. This information is useful in providing initial estimates of spacing VAWTs in a wind farm.

The airfoil section in a VAWT encounters a cyclically varying incident flowfield. Based on kinematics, the geometric angle of attack encountered by the airfoil section (as a function of the azimuthal rotation angle $\xi)$ is

$$
\alpha(\xi)=\tan ^{-1}\left[\frac{\sin \xi}{\cos \xi+\Omega}\right]
$$

where $\Omega$ is the tip speed ratio. This variation is shown in Fig. 9a for two representative tip speed ratios with the wind aligned with the $\mathrm{x}$ axis and the turbine axis aligned with the $\mathrm{z}$ axis. The sense of rotation is anticlockwise as shown in Fig. 2. The maximum geometric angle of attack is achieved on the retreating side of the blade, just beyond the $90^{\circ}$ azimuthal location. Given that the VAWT in question is largely a lift driven device, simple quasi-steady aerodynamics (ignoring the effects of virtual camber introduced by the 


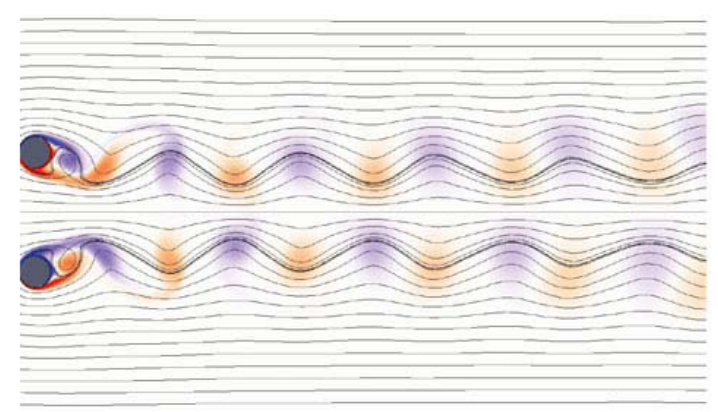

(a) Experimental, $\Omega=1.0$

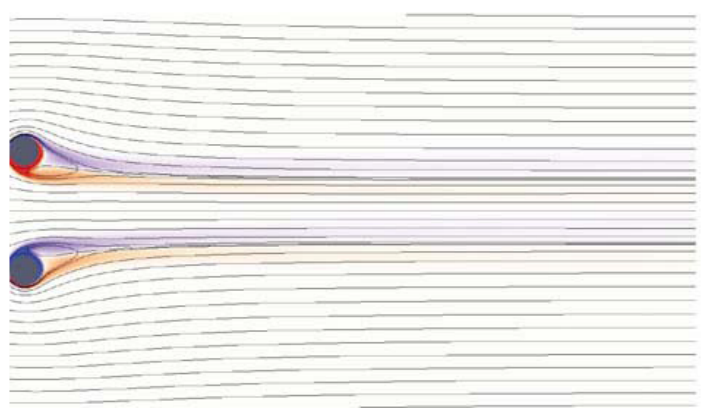

(c) Experimental, $\Omega=1.88$

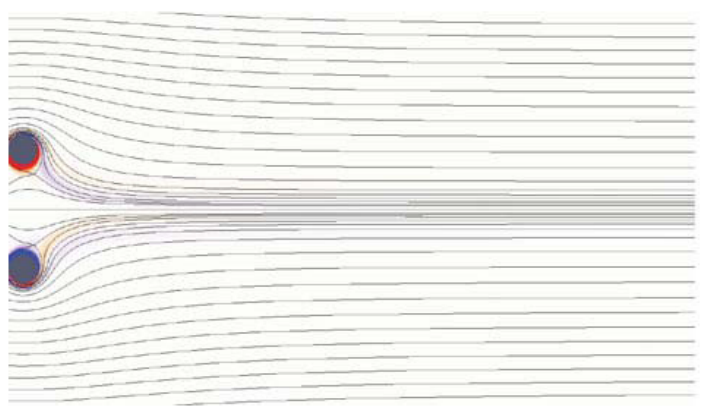

(e) Experimental, $\Omega=3.0$

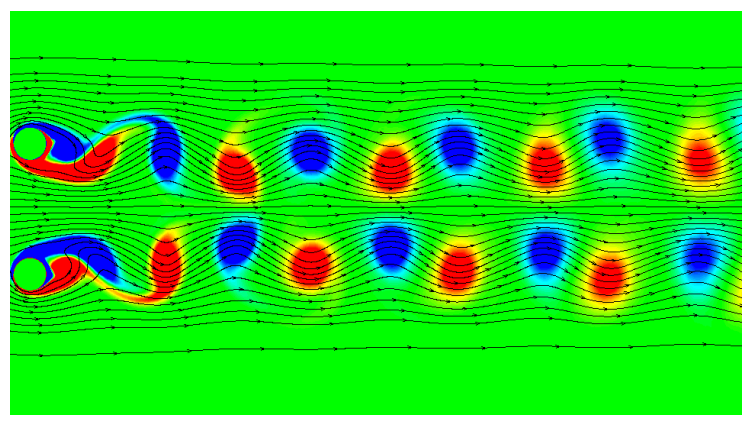

(b) Computational, $\Omega=1.0$

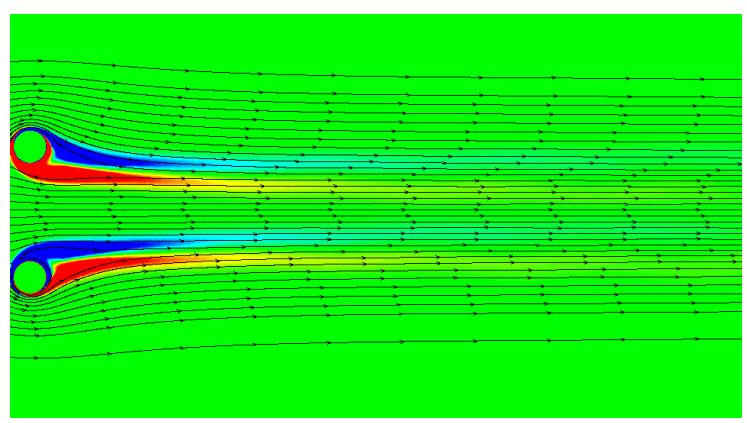

(d) Computational, $\Omega=1.88$

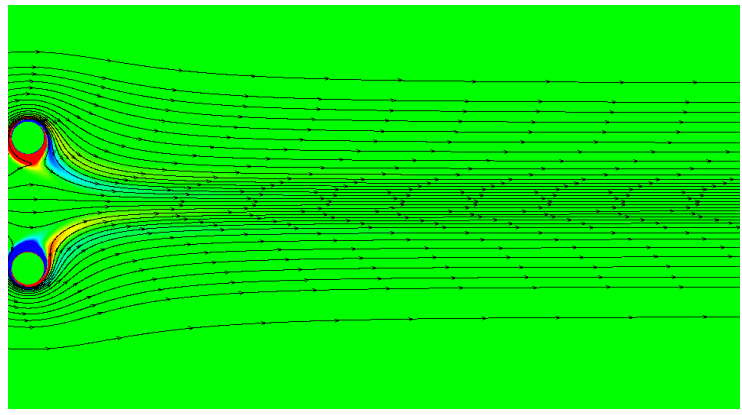

(f) Computational, $\Omega=3.0$

Figure 5. Streamlines and vorticity at different rotational speeds for the doublet-like counter-rotating cylinders.
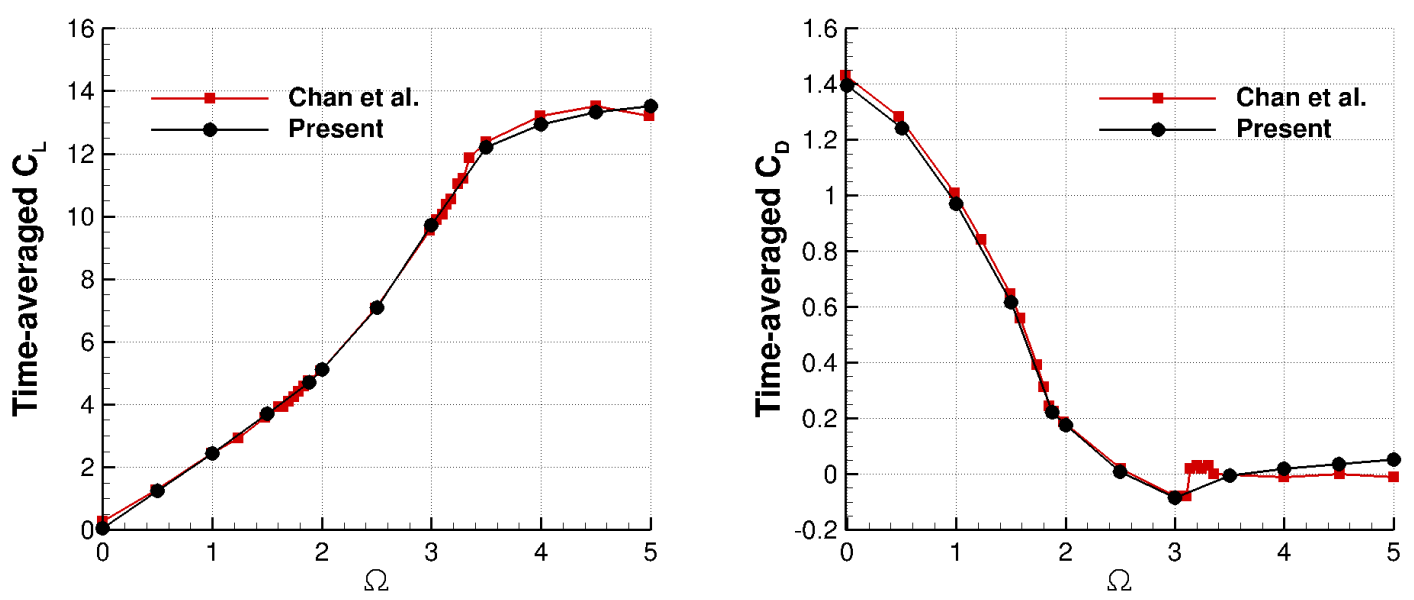

Figure 6. Time-averaged lift and drag coefficients for counter-rotating cylinders. 
non-uniform incident velocity field) gives the following result for the power coefficient of the airfoil:

$$
C_{P}=\frac{T \omega R}{\frac{1}{2} \rho_{\infty} D U_{\infty}^{3}}=2 \pi \alpha \sin \alpha \Omega \frac{c}{2 R}(\Omega+\cos \xi)^{2},
$$

where $T$ is the tangential force per unit span. A comparison of the power predicted by the quasi-steady model and actual computations is shown in Fig. 9b at TSR $=5.0$. The productive part of the cycle ranges between $50^{\circ}<\xi<150^{\circ}$ while the downstream half of the cycle is immersed in the shed wake system and does not generate useful power.

\section{A. Pairs of VAWTs}

Before investigating the performance of arrays of VAWTs, it is instructive to examine the characteristics of pairs of VAWTs. Two cases are considered in which the line joining the axis of the 2-bladed VAWTs is aligned perpendicular to the freestream wind. The vertical separation distance between the VAWT axes is $S_{y}=2.5 \mathrm{D}$. In the co-rotating case, both turbines are given a counter-clockwise rotation. In the counterrotating case, the turbine above the symmetry line is given a counter-clockwise rotation and the turbine below the symmetry line is given a clockwise rotation.

Figure 10 compares the power coefficient of one blade of an isolated VAWT with that of co-rotating and counter-rotating VAWT pairs. The coupled VAWTs exhibit an amplification in power, most pronounced near the $\xi=100^{\circ}$ azimuthal location. The additional power is generated by the velocity field induced by the neighboring turbine and can be understood from Fig. 11, which shows an airfoil section near the point of maximum power. Using a simple quasi-steady approximation, the lift force (per unit span) can be calculated as

$$
L\left(\xi=90^{\circ}\right) \approx 2 \pi\left[\frac{U_{\infty}+u_{i}+u_{o}}{\omega R}\right]\left[\frac{1}{2} \rho\left((\omega R)^{2}+\left(U_{\infty}+u_{i}+u_{o}\right)^{2}\right)\right] c,
$$

where $u_{i}$ is the self-induced velocity (in the streamwise direction) of the turbine and $u_{o}$ is the streamwise velocity induced by the net effect of the neighboring turbines. The tangential force coefficient (ignoring second order terms) is then

$$
C_{F_{t}}\left(\xi=90^{\circ}\right) \approx 2 \pi\left[\frac{1+2 \frac{u_{i}+u_{o}}{U_{\infty}}}{\Omega^{2}}\right]\left[\Omega^{2}+1+2 \frac{u_{i}+u_{o}}{U_{\infty}}\right] \frac{c}{2 R} .
$$

Note that the first term in the square brackets is the contribution due to the effective angle of attack and the second term is due to the dynamic pressure. Given that $\Omega^{2}$ can be expected to be much larger than $\left(u_{i}+u_{o}\right) / U_{\infty}$, it is clear that the major contributor to the improved performance is the increased angle of attack (and hence a larger tilt of the force vector toward the tangential direction).

\section{B. Arrays of VAWTs}

Figure 12 shows the time averaged wake of an infinite column of co-rotating 2-bladed VAWTs whose axes are located on the y axis and separated by a vertical distance $S_{y}=2.5 D$ for three different TSRs. Similar to in isolated VAWT, the spatial extent of the wake deficit diminishes with increased rotational speed, however the wake deficit region is seen to extend further downstream when compared to the isolated turbine situation. The interference effects between the turbines gives rise to regions of substantial excess momentum between the turbines. The resulting induced velocities contribute to an augmentation in the generated power (Fig. 13), similar to that seen in the previous section. A VAWT operating in a column of co-rotating VAWTs provides $50 \%--100 \%$ power augmentation compared to an isolated VAWT, depending on the rotational speed.

Figure 14 shows the time averaged wake of 5 columns of co-rotating 2-bladed VAWTs that are vertically separated by a distance $S_{y}=2.5 \mathrm{D}$. Three different TSRs and two different horizontal spacings, $S_{x}=3.0$ and $S_{x}=4.0$, are considered. Very high levels of excess momentum can be observed in between the downstream columns and the wake deficit region is also seen to reduce in extent for the downstream columns. The instantaneous power coefficient for one blade in each column is shown in Figures 15, 16 and a high level of unsteadiness as well as amplification of power is evident. Figure 17 compares the time averaged and peak-to-peak power coefficients over ten revolutions. While the first column produces slightly less power 


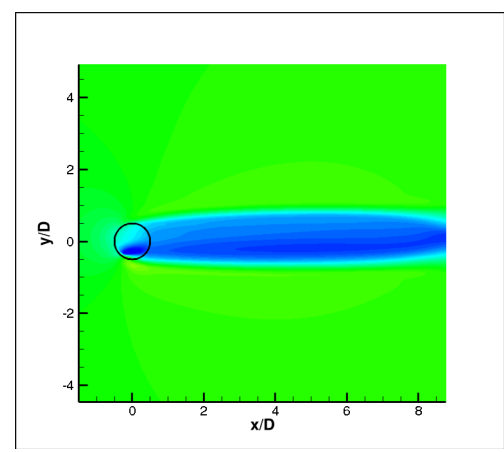

(a) $\mathrm{TSR}=2.5,2$ blades

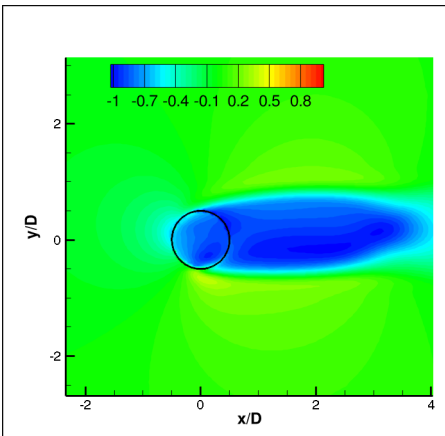

(d) $\mathrm{TSR}=2.5,4$ blades

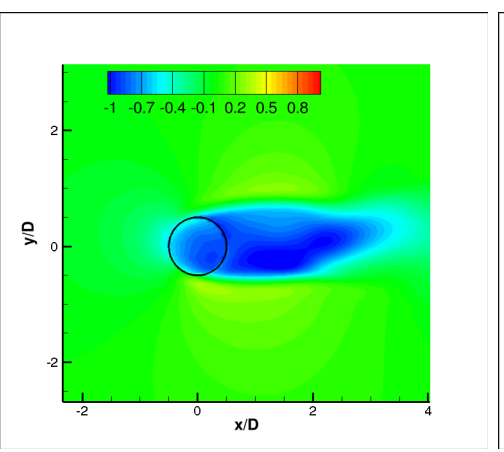

(b) $\mathrm{TSR}=5.0,2$ blades

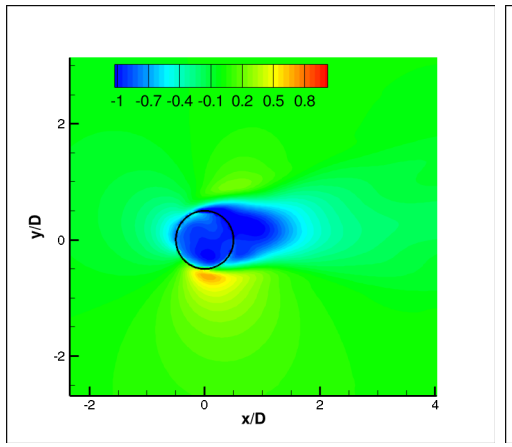

(e) $\mathrm{TSR}=5.0,4$ blades

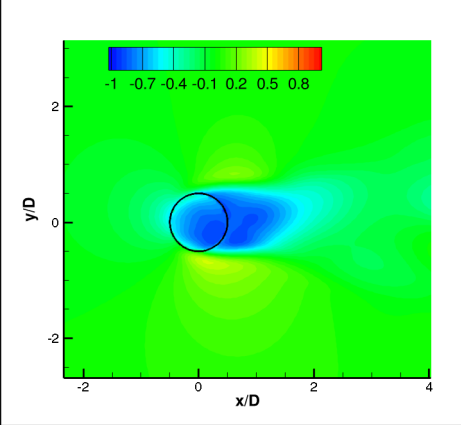

(c) $\mathrm{TSR}=7.5,2$ blades

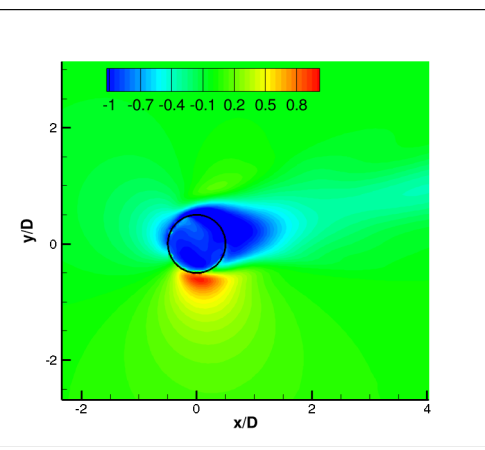

(f) $\mathrm{TSR}=7.5,4$ blades

Figure 7. Time averaged flow over a VAWT: $\left(U-U_{\infty}\right) / U_{\infty}$.

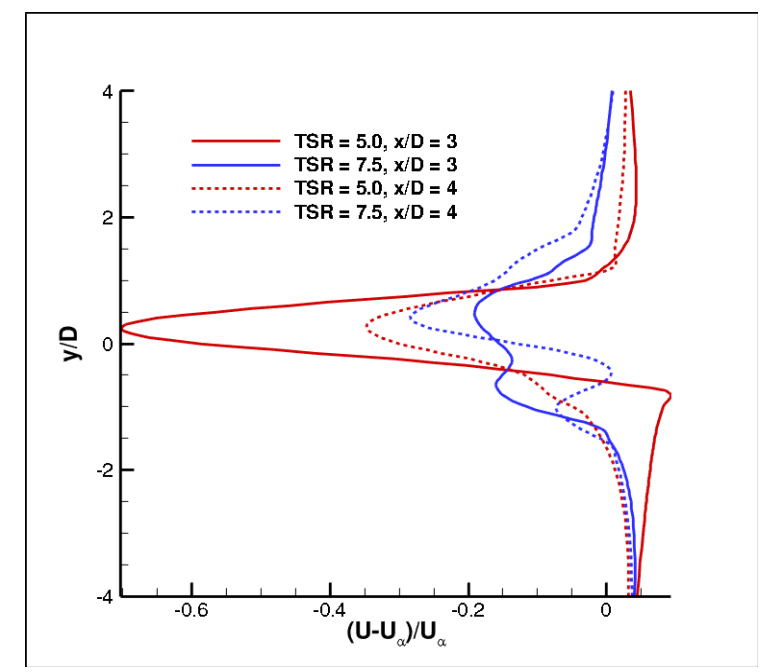

Figure 8. Time averaged flow over a 2-bladed VAWT: $\left(U-U_{\infty}\right) / U_{\infty}$ at selected streamwise locations.

than the single array of VAWTS, in certain configurations (Figure 17e), the downstream turbines are seen to produce more power than the upstream ones. The power production capability of downstream turbines also shows a strong dependence on the horizontal separation of the columns.

Similar benefits are noticeable when alternate columns of VAWTs are counter-rotating (Figures 18, 19). Having alternate columns of counter-rotating turbines can be highly beneficial or detrimental as evidenced in the power production of the second and third columns (Fig. 19e). For the second column, the power producing part of the cycle benefits from the excess momentum region, whereas for the third column, the productive part of the cycle lies directly in the wake of the preceding column. The drastic difference in performance in the various arrangements discussed above highlights the potential for optimization of turbine 

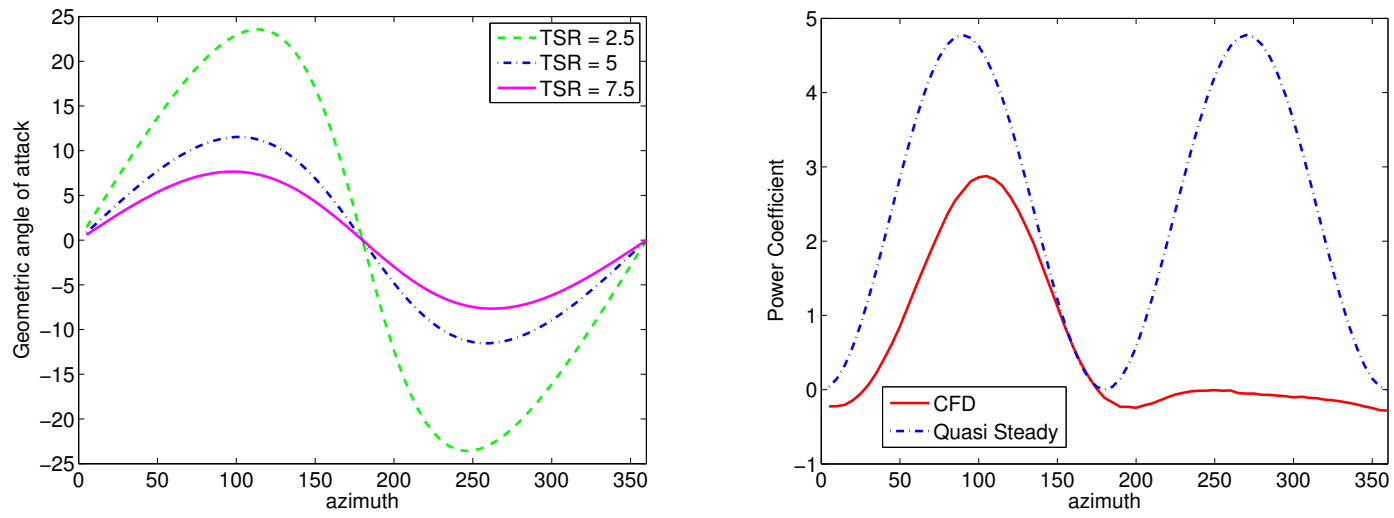

Figure 9. Quasi-steady approximation for a VAWT.
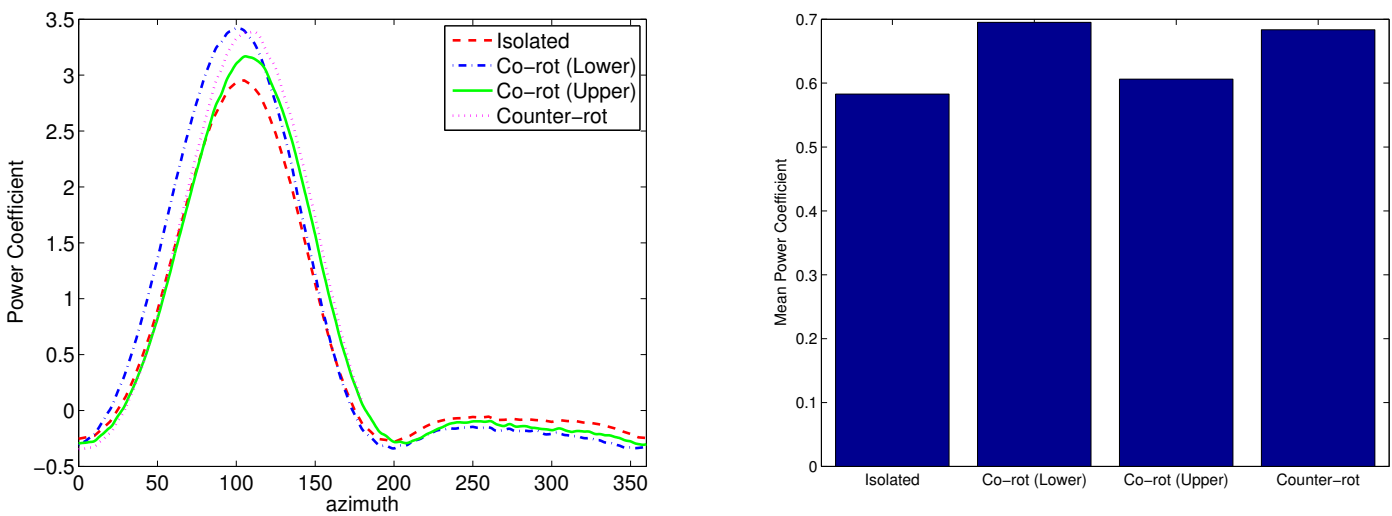

Figure 10. Power Coefficient of an isolated VAWT compared with that of co-rotating and counter-rotating VAWT pairs operating at $\mathrm{TSR}=\mathbf{5 . 0}$.

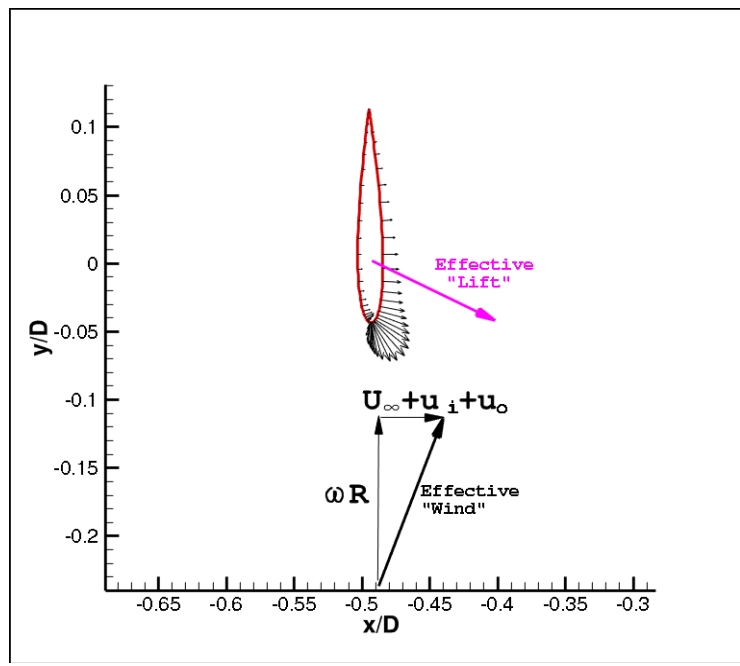

Figure 11. Schematic of the incident velocity field and forces on a VAWT airfoil section.

placement. 


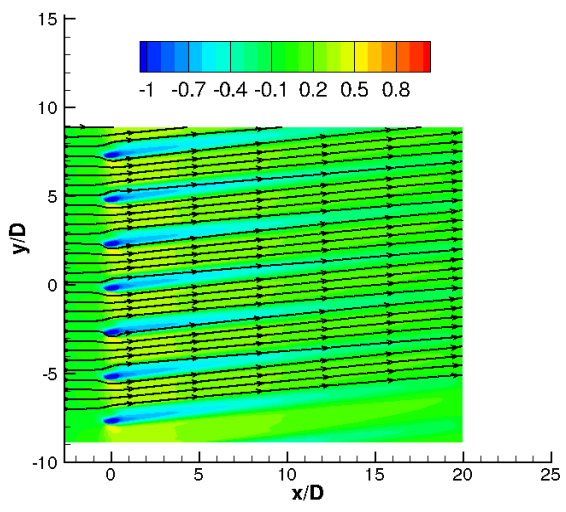

(a) $\mathrm{TSR}=2.5$

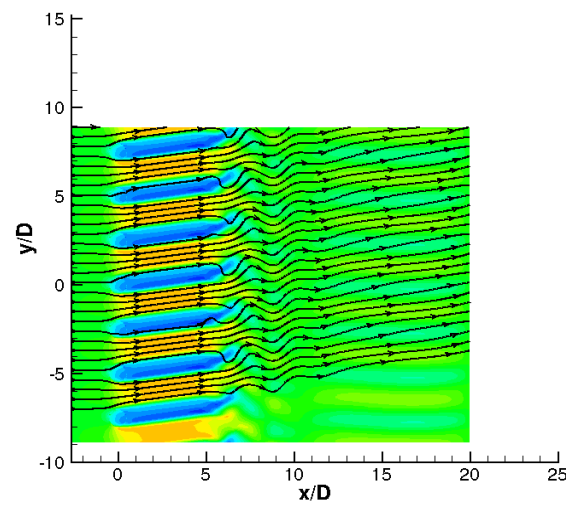

(b) $\mathrm{TSR}=5.0$

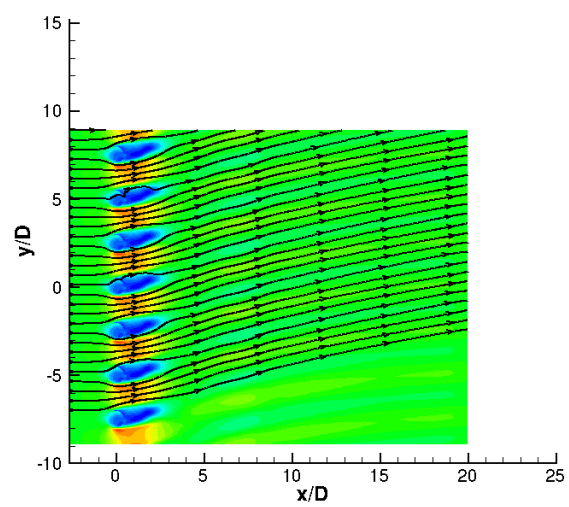

(c) $\mathrm{TSR}=7.5$

Figure 12. Time averaged flow over a co-rotating VAWT column: $\left(U-U_{\infty}\right) / U_{\infty}$.

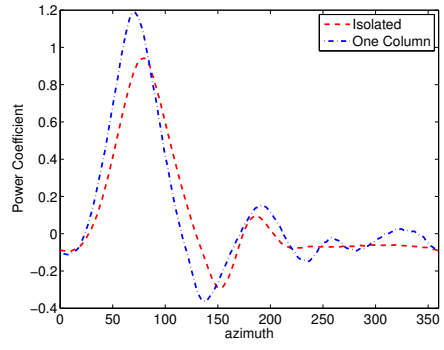

(a) $\mathrm{TSR}=2.5$

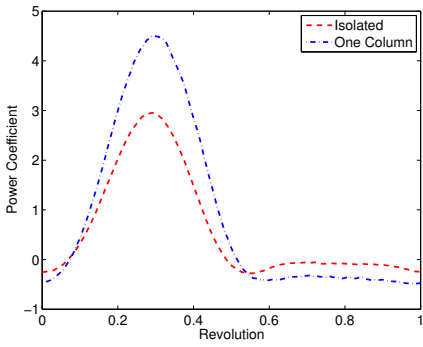

(b) $\mathrm{TSR}=5.0$

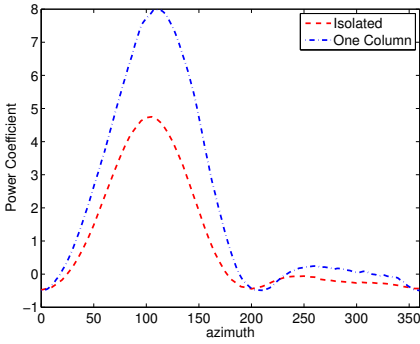

(c) $\mathrm{TSR}=7.5$

Figure 13. Power Coefficient for one blade of a co-rotating VAWT column.

\section{Conclusions}

The performance of isolated, pairs, columns and arrays of Vertical Axis Wind Turbines (VAWTs) was analyzed numerically with an emphasis on the underlying fluid dynamic phenomena. An unsteady Reynolds Averaged Navier Stokes solver was employed in this study and validations were presented on rotating cylinder and VAWT configurations. For isolated VAWTs, the majority of the wake deficit is confined to a few diameters downstream and the spatial extent of the deficit is seen to diminish with increased rotational speed. Pairs of co-rotating or counter-rotating VAWTs were seen to generate more power than an isolated VAWT by taking advantage of the beneficial induced velocity field. A column of co-rotating VAWTs was seen 
to provide between $50 \%-100 \%$ of power augmentation compared to an isolated VAWT. The interference effects between VAWTs results in regions of momentum in excess to that of the freestream. It was shown that in an array of VAWTs, downstream turbines can be more efficient than upstream turbines under optimal conditions.

Overall, this work is of a preliminary nature and highlights the potential to manage and harness wake regions in VAWT arrays to achieve high wind farm efficiencies. Continuing and future work will focus on practical considerations such as self-driven turbines and lower tip speed ratios. Further, seeking robust solutions in the presence of varying wind directions may necessitate the investigation of staggered arrangement of turbines and scheduling of rotational speeds.

\section{Acknowledgments}

The authors acknowledge Prof. John Dabiri for several fruitful discussions and insightful comments.

\section{References}

${ }^{1}$ Dabiri, J., "Potential order-of-magnitude enhancement of wind farm power density via counter-rotating vertical-axis wind turbine arrays," Journal of Renewable and Sustainable Energy, Vol. 3, 2011.

${ }^{2}$ Sorenson, V., "Renewable Energy: Its Physics, Engineering, Use, Environmental Impacts, Economy, and Planning Aspects," Elsevier, London, 2004.

${ }^{3}$ MacKay, D., "Sustainable EnergyWithout the Hot Air," UIT Cambridge Ltd., Cambridge, UK, 2009.

${ }^{4}$ Whittlesey, R., Liska, S., and Dabiri, J., "Fish schooling as a basis for vertical-axis wind turbine farm design," Bioinspiration and Biomimetics, Vol. 5, 2010.

${ }^{5}$ Duraisamy, K., Ramasamy, M., Baeder, J., and Leishman, G., "High Resolution Wake Capturing Methodology for Hovering Rotor Simulations," Journal of the American Helicopter Society, Vol. 52, No. 2, 2007.

${ }^{6}$ Lakshminarayan, V. K., "Computational Investigation of Micro-Scale Coaxial Rotor Aerodynamics in Hover," Ph.D. dissertation, Department of Aerospace Engineering, 2009.

${ }^{7}$ Turkel, E., "Preconditioning Techniques in Computational Fluid Dynamics," Annual Review of Fluid Mechanics, Vol. 31, 1999, pp. 385-416.

${ }^{8}$ Spalart, P. and Allmaras, S., "A one-equation turbulence model for aerodynamic flows," AIAA, Aerospace Sciences Meeting and Exhibit, 30 th, Reno, NV, 1992.

${ }^{9}$ Yang, K., Lakshminarayan, V., and Baeder, J., "Simulation of a Cycloidal Rotor System Using an Overset RANS Solver," American Helicopter Society 66th Annual Forum Proceedings, Phoenix, AZ, May 2010.

${ }^{10}$ Benedict, M., Jarugumilli, T., Lakshminarayan, V., and Chopra, I., "Effect of Flow Curvature on the Forward Flight Performance of a MAV-Scale Cycloidal Rotor," In press AIAA journal.

${ }^{11}$ Jarugumilli, T., Lind, A., Benedict, M., Lakshminarayan, V., Jones, A., and Chopra, I., "Experimental and Computational Flow Field Studies of a MAV-scale Cycloidal Rotor in Forward Flight," American Helicopter Society 69th Annual Forum Proceedings, Phoenix, AZ, May 2013.

${ }^{12}$ Benedict, M., Lakshminarayan, V., Pino, J., and Chopra, I., "Fundamental Understanding of the Physics of a SmallScale Vertical Axis Wind Turbine with Dynamic Blade Pitching: An Experimental and Computational Approach," 54th AIAA Structural Dynamics, and Materials Conference, Boston, MA, April 2013.

${ }^{13}$ Oler, J., Strickland, J., Im, B., and Graham, G., "Dynamic Stall Regulation of the Darrieus Turbine," Tech. Rep. SAND83-7029, Sandia National Laboratories, Albuquerque, New Mexico 87185 and Livermore, California $94550,1983$.

${ }^{14}$ Chan, A., Dewey, P., Jameson, A., Liang, C., and Smits, A., "Vortex suppression and drag reduction in the wake of counter-rotating cylinders," Journal of Fluid Mechanics, Vol. 679, 2011, pp. 343-383. 


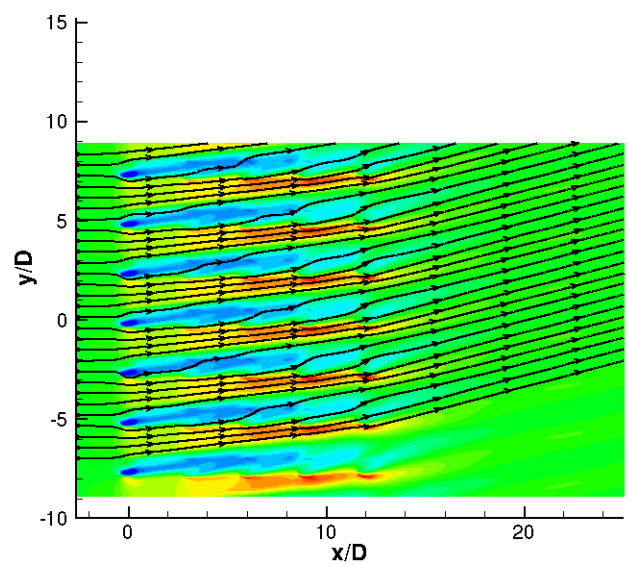

(a) $\mathrm{TSR}=2.5, S_{x} / D=3$

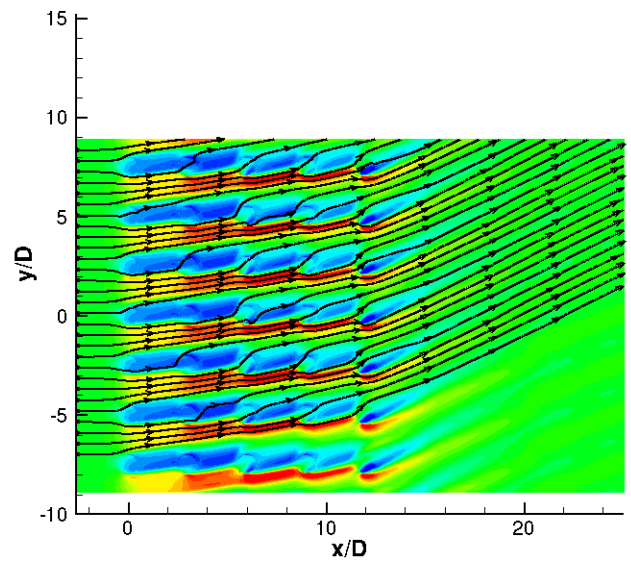

(c) $\mathrm{TSR}=5.0, S_{x} / D=3$

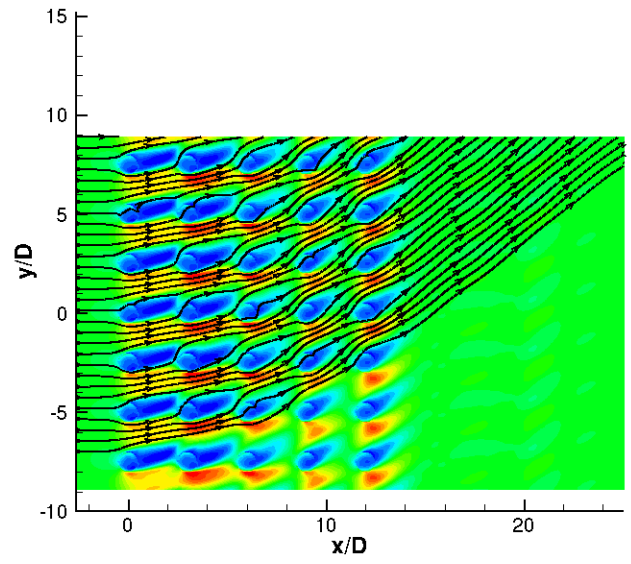

(e) $\mathrm{TSR}=7.5, S_{x} / D=3$

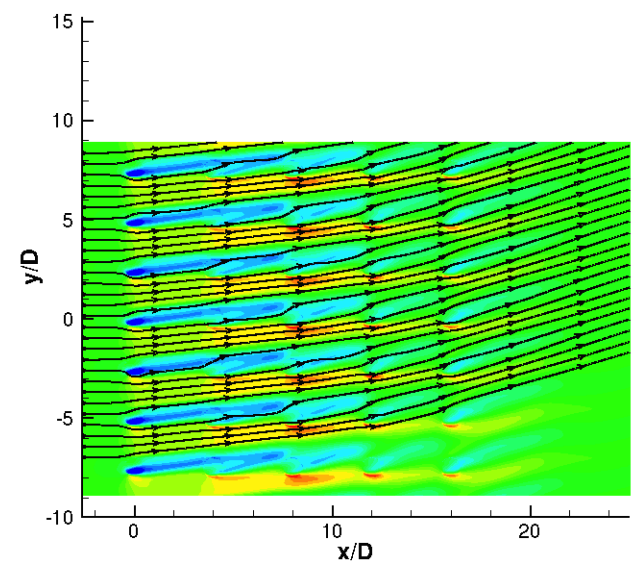

(b) $\mathrm{TSR}=2.5, S_{x} / D=4$

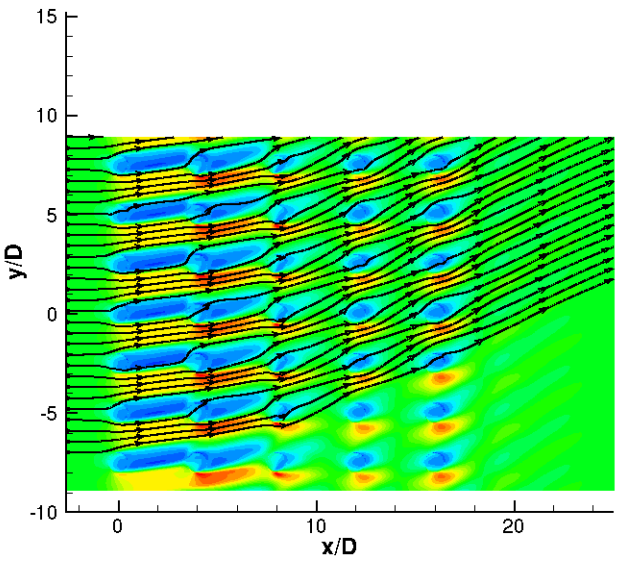

(d) $\mathrm{TSR}=5.0, S_{x} / D=4$

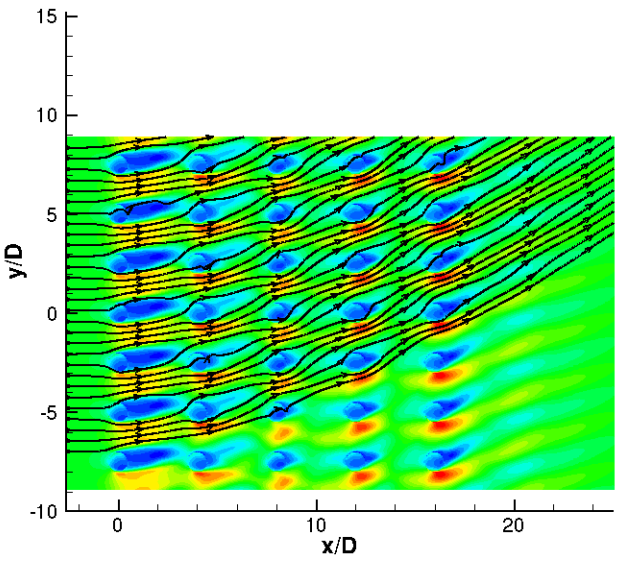

(f) $\mathrm{TSR}=7.5, S_{x} / D=4$

Figure 14. Time averaged flow over a 5 column co-rotating VAWT array: $\left(U-U_{\infty}\right) / U_{\infty}$. Contour labels as in Fig. 12. 


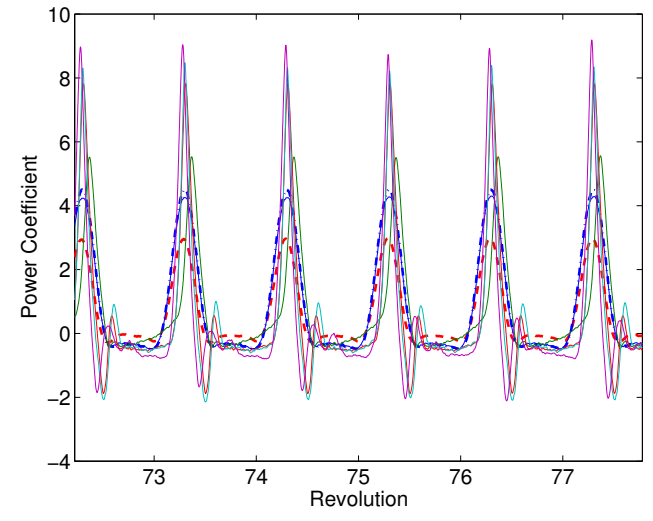

(a) $\mathrm{TSR}=5.0, S_{x} / D=3$

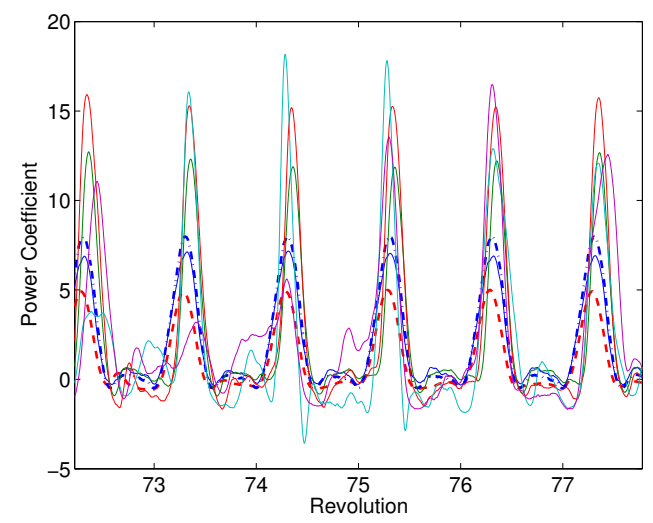

(c) $\mathrm{TSR}=7.5, S_{x} / D=3$

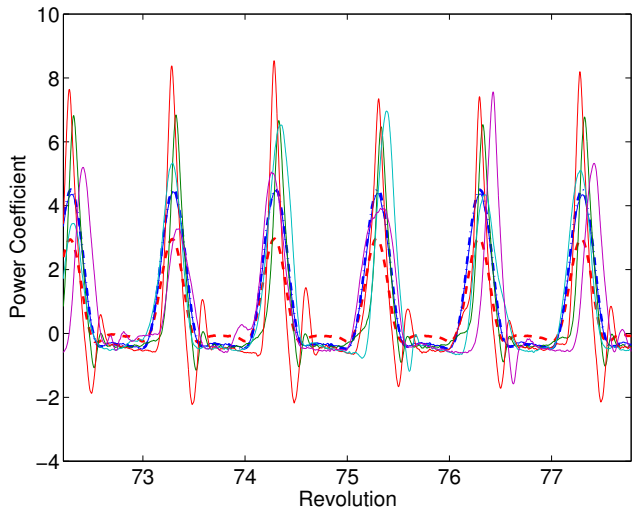

(b) $\mathrm{TSR}=5.0, S_{x} / D=4$

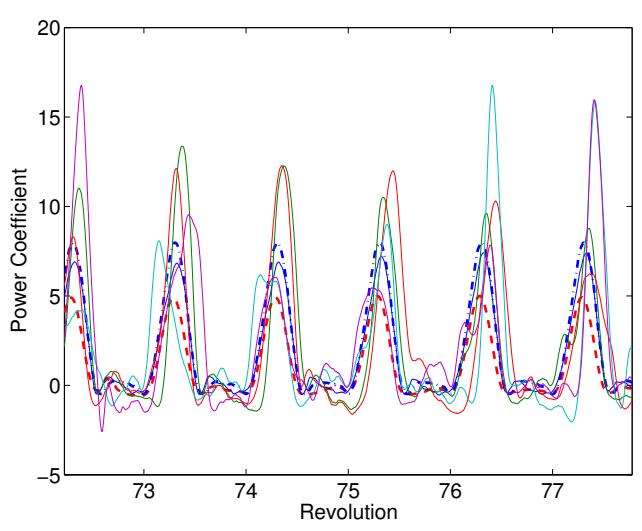

(d) $\mathrm{TSR}=7.5, S_{x} / D=4$

Figure 15. Instantaneous power coefficient generated by different blades of a 5 column co-rotating VAWT array. 


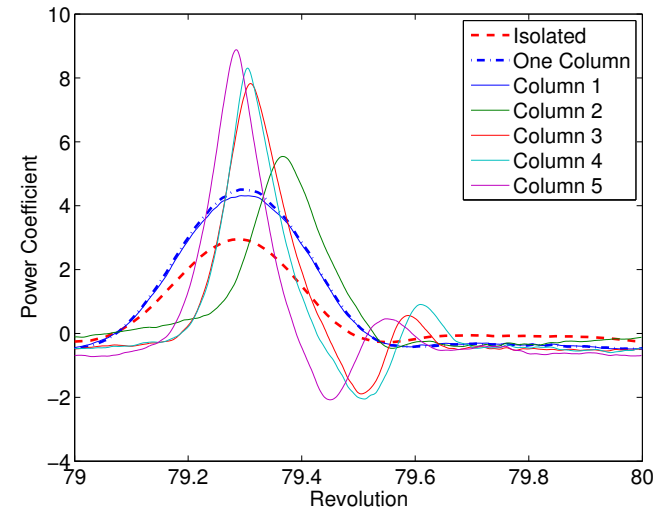

(a) $\mathrm{TSR}=5.0, S_{x} / D=3$

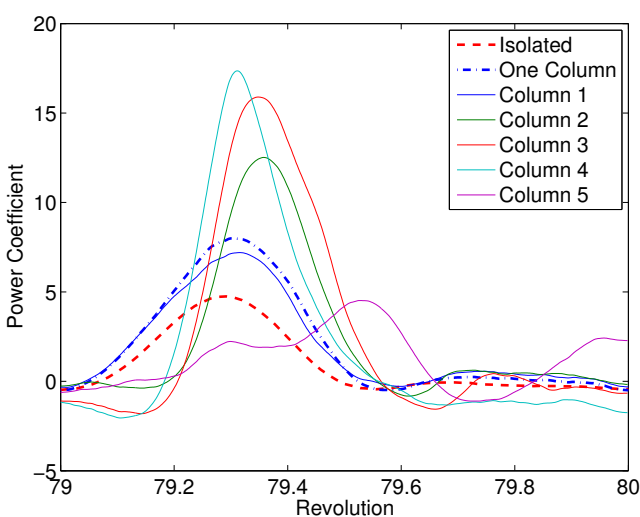

(c) $\mathrm{TSR}=7.5, S_{x} / D=3$

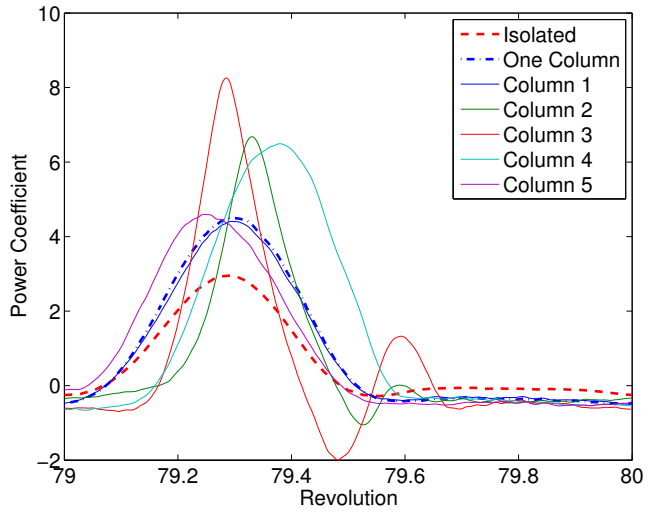

(b) $\mathrm{TSR}=5.0, S_{x} / D=4$

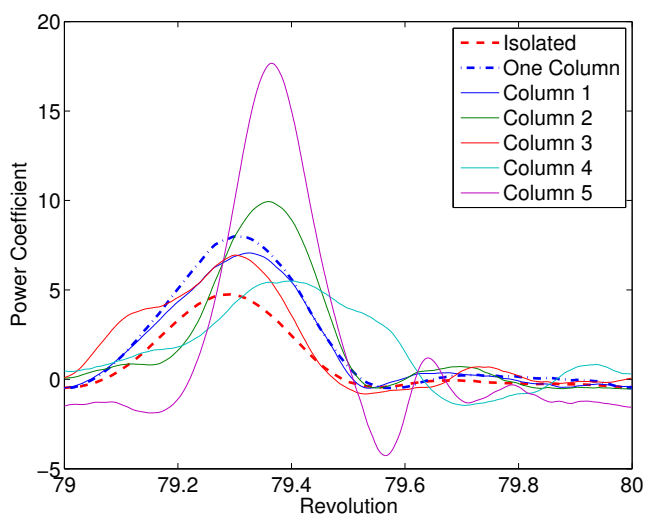

(d) $\mathrm{TSR}=7.5, S_{x} / D=4$

Figure 16. Instantaneous power coefficient generated by different blades of a 5 column co-rotating VAWT array. 


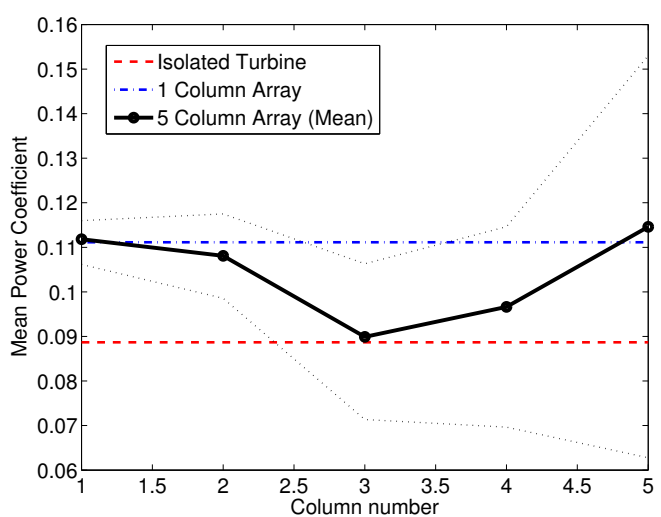

(a) $\mathrm{TSR}=2.5, S_{x} / D=3$

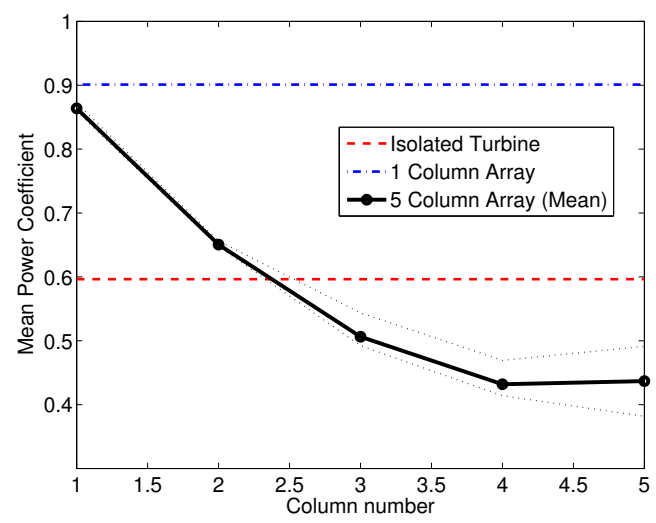

(c) $\mathrm{TSR}=5.0, S_{x} / D=3$

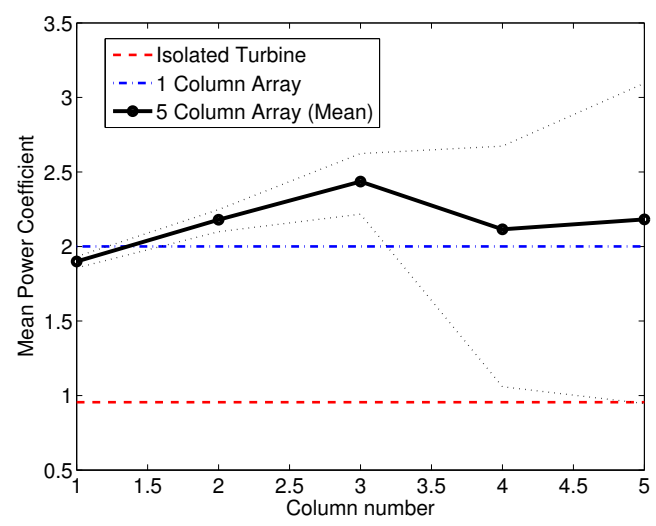

(e) $\mathrm{TSR}=7.5, S_{x} / D=3$

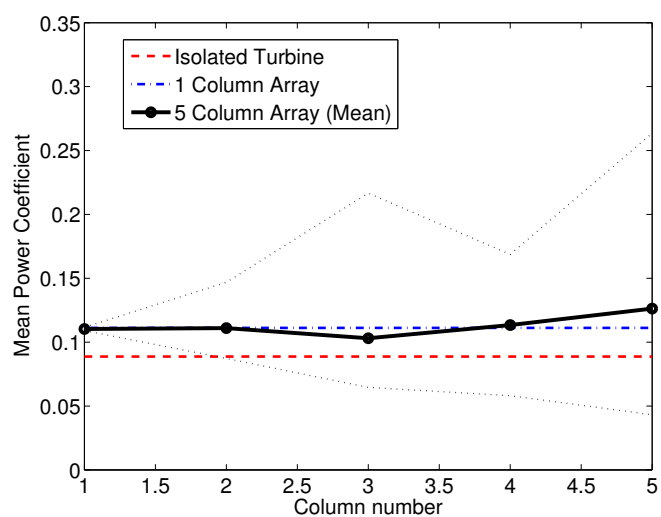

(b) $\mathrm{TSR}=2.5, S_{x} / D=4$

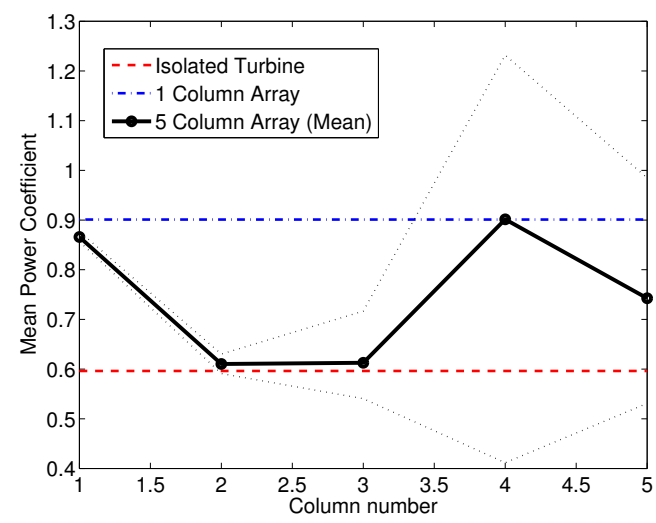

(d) $\mathrm{TSR}=5.0, S_{x} / D=4$

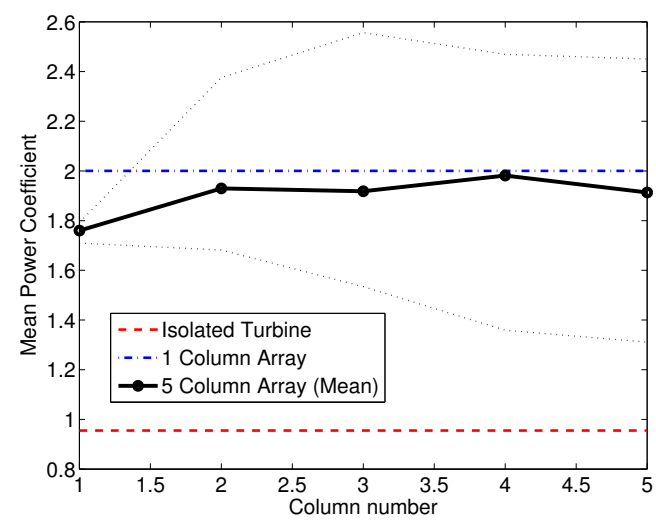

(f) $\operatorname{TSR}=7.5, S_{x} / D=4$

Figure 17. Mean power coefficient generated by different blades of a 5 column co-rotating VAWT array. Black dotted lines depict max and min variability in periodically averaged power coefficient. 


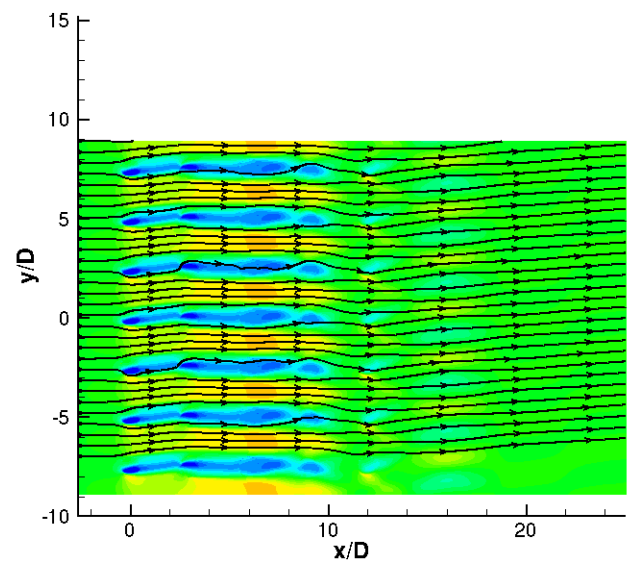

(a) $\mathrm{TSR}=2.5, S_{x} / D=3$

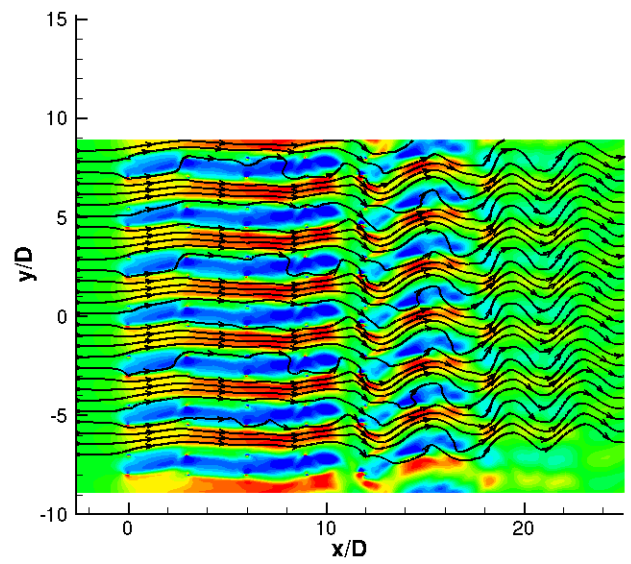

(c) $\operatorname{TSR}=5.0, S_{x} / D=3$

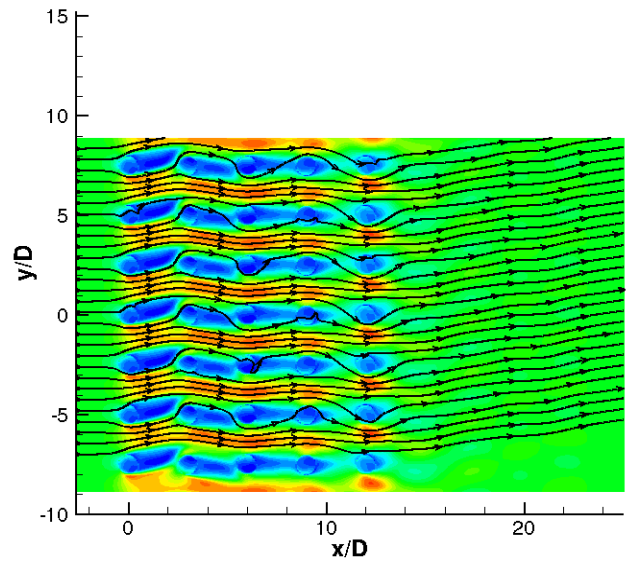

(e) $\mathrm{TSR}=7.5, S_{x} / D=3$

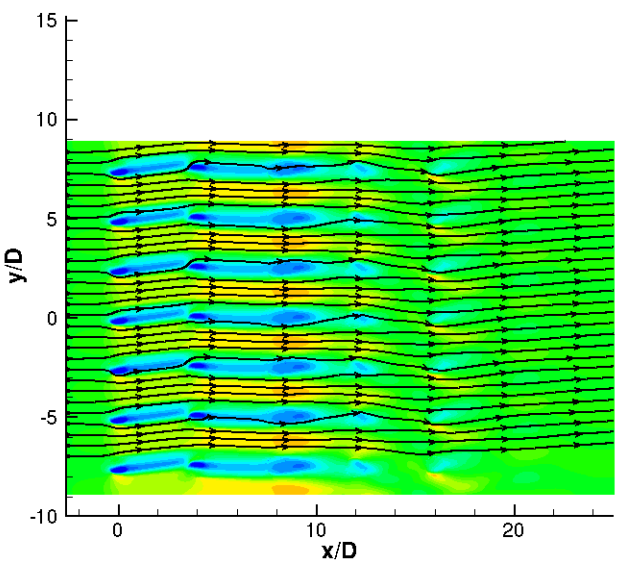

(b) $\mathrm{TSR}=2.5, S_{x} / D=4$

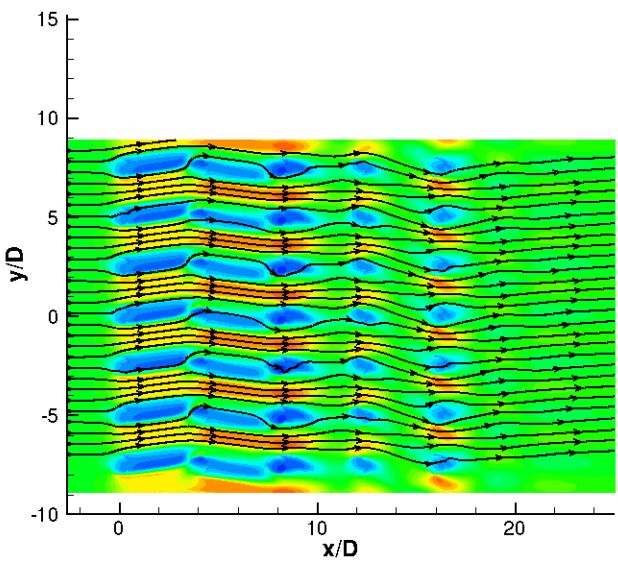

(d) $\mathrm{TSR}=5.0, S_{x} / D=4$

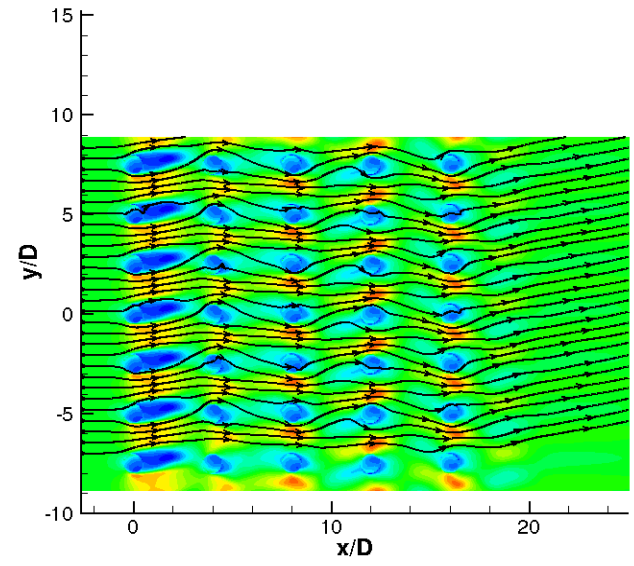

(f) $\mathrm{TSR}=7.5, S_{x} / D=4$

Figure 18. Time averaged flow over a 5 column VAWT array with alternate columns counter-rotating: $(U-$ $\left.U_{\infty}\right) / U_{\infty}$. Contour labels as in Fig. 12. 


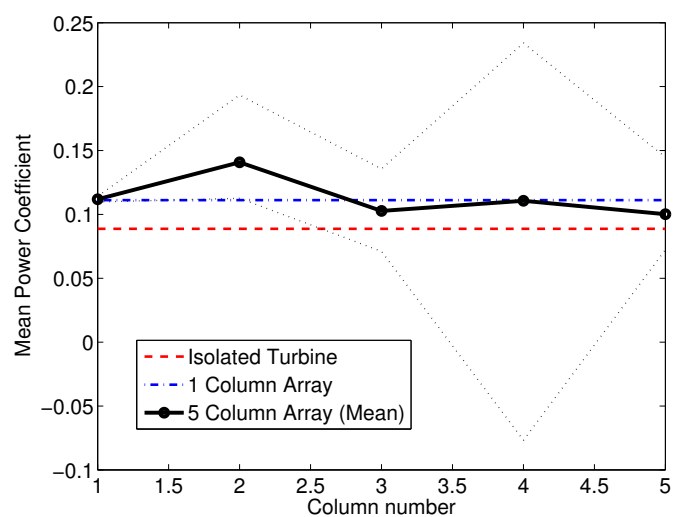

(a) $\mathrm{TSR}=2.5, S_{x} / D=3$

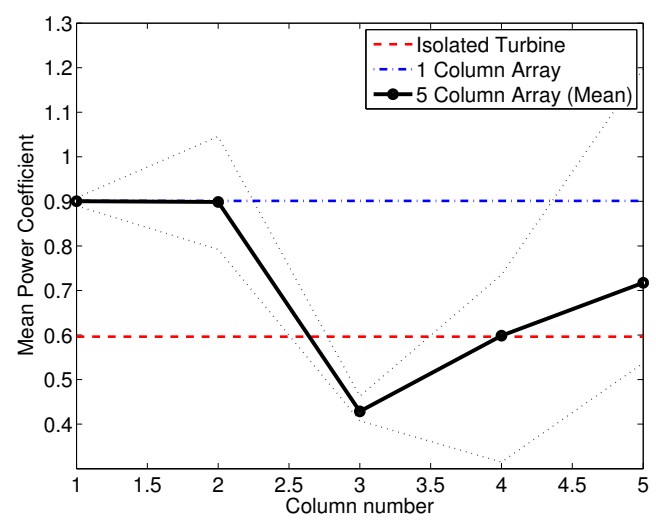

(c) $\mathrm{TSR}=5.0, S_{x} / D=3$

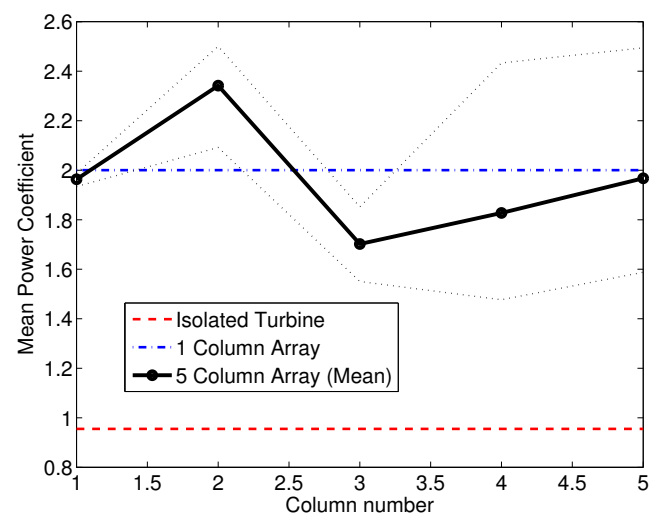

(e) $\mathrm{TSR}=7.5, S_{x} / D=3$

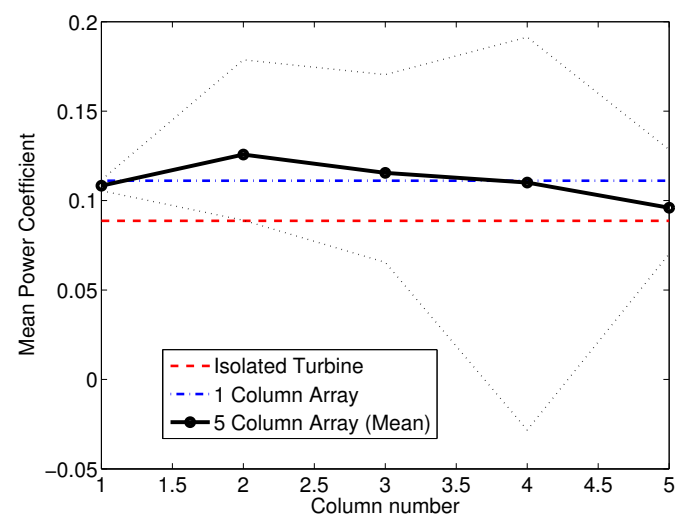

(b) $\mathrm{TSR}=2.5, S_{x} / D=4$

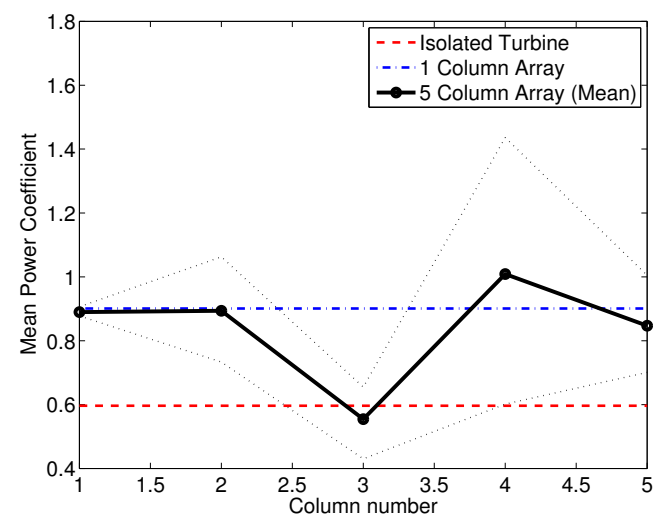

(d) $\mathrm{TSR}=5.0, S_{x} / D=4$

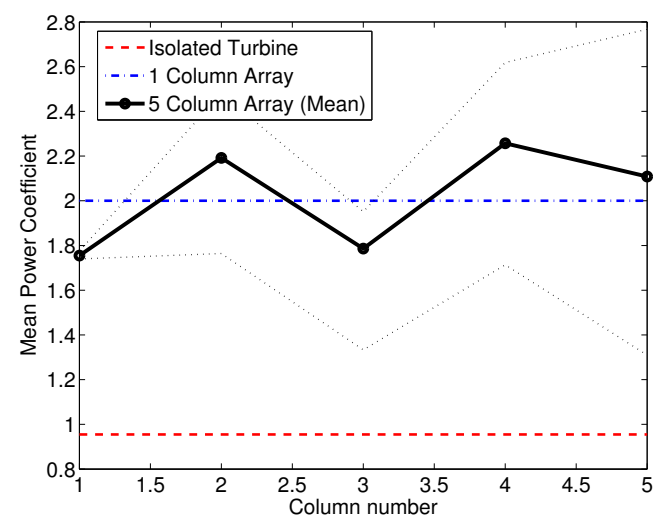

(f) $\mathrm{TSR}=7.5, S_{x} / D=4$

Figure 19. Mean power coefficient generated by different blades of a 5 column VAWT array with alternate columns counter rotating. 